Nonlinear Processes in Geophysics (2005) 12: 245-255

SRef-ID: $1607-7946 / \mathrm{npg} / 2005-12-245$

European Geosciences Union

(c) 2005 Author(s). This work is licensed

under a Creative Commons License.

\title{
Intermittency in MHD turbulence and coronal nanoflares modelling
}

\author{
P. Veltri ${ }^{1}$, G. Nigro ${ }^{1}$, F. Malara ${ }^{1}$, V. Carbone ${ }^{1}$, and A. Mangeney ${ }^{2}$ \\ ${ }^{1}$ Dipartimento di Fisica, Università della Calabria, I-87030 Arcavacata di Rende, Italy \\ ${ }^{2}$ LESIA - Observatoire de Paris, Section de Meudon 5, place Jules Janssen, 92195 Meudon Cedex, France
}

Received: 15 September 2004 - Revised: 27 January 2005 - Accepted: 28 January 2005 - Published: 9 February 2005

Part of Special Issue "Advances in space environment turbulence"

\begin{abstract}
High resolution numerical simulations, solar wind data analysis, and measurements at the edges of laboratory plasma devices have allowed for a huge progress in our understanding of MHD turbulence. The high resolution of solar wind measurements has allowed to characterize the intermittency observed at small scales. We are now able to set up a consistent and convincing view of the main properties of MHD turbulence, which in turn constitutes an extremely efficient tool in understanding the behaviour of turbulent plasmas, like those in solar corona, where in situ observations are not available. Using this knowledge a model to describe injection, due to foot-point motions, storage and dissipation of MHD turbulence in coronal loops, is built where we assume strong longitudinal magnetic field, low beta and high aspect ratio, which allows us to use the set of reduced MHD equations (RMHD). The model is based on a shell technique in the wave vector space orthogonal to the strong magnetic field, while the dependence on the longitudinal coordinate is preserved. Numerical simulations show that injected energy is efficiently stored in the loop where a significant level of magnetic and velocity fluctuations is obtained. Nonlinear interactions give rise to an energy cascade towards smaller scales where energy is dissipated in an intermittent fashion. Due to the strong longitudinal magnetic field, dissipative structures propagate along the loop, with the typical speed of the Alfvén waves. The statistical analysis on the intermittent dissipative events compares well with all observed properties of nanoflare emission statistics. Moreover the recent observations of non thermal velocity measurements during flare occurrence are well described by the numerical results of the simulation model. All these results naturally emerge from the model dynamical evolution without any need of an adhoc hypothesis.
\end{abstract}

Correspondence to: P. Veltri

(veltri@ fis.unical.it)

\section{Introduction}

In the usual picture of turbulence, in an incompressible fluid, described by Navier-Stokes equations, when Reynolds number $R=(V L) / \nu \gg 1(V$ and $L$ are typical values for the fluid velocity and length scale, while $v$ is the kinematic viscosity) nonlinear terms prevail with respect to dissipative terms, giving rise to a nonlinear energy cascade from large to small length scales. Three ranges of lengths $\ell$ can be identified: an injection range $(\ell \simeq L)$, an inertial range where energy is transferred towards smaller and smaller lengths $(\eta \ll \ell \ll L)$, and a dissipation range $(\ell \simeq \eta)$ where viscosity becomes the dominant physical effect.

Due to the lack of any characteristic length in the inertial range, the turbulent energy cascade is usually considered a self similar, fractal process consisting in a hierarchy of eddies of different length scale. Actually, turbulence is intermittent, in that it does not display a fractal, but rather a multifractal behavior, whose properties are determined by the topology of the most intermittent structures which are found at the smallest lengths. The identification of these most intermittent structures represents then a crucial problem in the framework of turbulence.

In the last years, solar wind has offered us a unique opportunity to study turbulence, and in particular MHD turbulence. In fact solar wind represents a supersonic and superalfvénic flow, from where space experiments have given a wealth of data (velocity, magnetic field, plasma density, temperature etc. or also particle distribution functions) at a resolution which is not available in any earth laboratory.

In the following section we will recast the results obtained when trying to identify the small scale coherent intermittent structures by analyzing velocity and magnetic field fluctuations in solar wind. In particular we will show that current sheet are naturally produced by MHD turbulence as coherent intermittent small scale structures. We will use these results to set up an efficient model describing the physical mechanism underlying coronal nanoflares. 
Solar flares represent impulsive energy releases in the solar corona. The released energy is observed in various forms: thermal, soft and hard x-ray emission, accelerated particles, etc. (Priest, 1982). The energy associated with each event is estimated to range from $\sim 10^{32} \mathrm{erg}$ down to $\sim 10^{23}-10^{24} \mathrm{erg}$. Statistical analysis of various quantities (energy, peak emission, duration time), characterizing the impulsive events, have shown that the probability distribution for these quantities, approximately follows a power law. When considering the energy distribution, the power law index is $\alpha \simeq 1.5-1.6$ in active regions, while $\alpha \simeq 2.04-2.5$ in the quiet corona (Lin et al., 1984; Crosby et al., 1993). More recently Boffetta et al. (1999) have stressed the fact that the probability distribution of time separation between two successive flares also displays power law (see also Lepreti et al., 2001, with index $\alpha \simeq 2.4$ ).

Parker (1988) conjectured that the smallest flares $\left(\sim 10^{24}\right.$ erg) ("nanoflares") should essentially be due to dissipation of many small current sheets, eventually producing intense magnetic dissipation in association with magnetic reconnection.

We will slightly modify Parker's conjecture by suggesting that nanoflares correspond to dissipation of many small current sheets generated in the nonlinear cascade occuring inside coronal magnetic structure in consequence of the power input in the form of Alfvén waves due to footpoint motion. In this view, current sheets are the naturally produced coherent intermittent small scale structures of MHD "turbulent energy cascade".

\section{Intermittent structures in MHD turbulence}

\subsection{Self similarity of turbulent fluctuations}

Kolmogorov (1941), using dimensional arguments, conjectured that, in the inertial range, the rms of velocity difference fluctuations $\delta v_{\ell}=v(r+\ell)-v(r)$ scales as

$$
<\left(\delta v_{\ell}\right)^{2}>^{1 / 2} \propto \ell^{\frac{1}{3}} \text {. }
$$

The lack of any characteristic length in the inertial domain implies that the nonlinear turbulent energy cascade is a self similar (fractal) process. Then, the probability distribution function for fluctuations $P\left(\delta v_{\ell}\right)$ should be invariant under the scale change

$P\left(\delta v_{\ell}\right)=\ell^{-\frac{1}{3}} F\left(\frac{\delta v_{\ell}}{\ell^{\frac{1}{3}}}\right)$,

which in turn only means that probability distribution functions of normalized velocity increments at different scales collapse on the same shape.

If Eq. (2) were valid, structure functions defined as

$S_{n}(\ell)=<\left|\delta v_{\ell}\right|^{n}>$,

i.e. higher order moments of velocity fluctuations, should scale as

$$
S_{n}(\ell)=\int_{-\infty}^{+\infty}\left|\delta v_{\ell}\right|^{n} P\left(\delta v_{\ell}\right) d\left(\delta v_{\ell}\right)=
$$

$\ell^{\frac{n}{3}} \int_{-\infty}^{+\infty}\left|\frac{\delta v_{\ell}}{\ell^{\frac{1}{3}}}\right|^{n} F\left(\frac{\delta v_{\ell}}{\ell^{\frac{1}{3}}}\right) d\left(\frac{\delta v_{\ell}}{\ell^{\frac{1}{3}}}\right)=C_{n} \ell^{\frac{n}{3}}$.

Structure functions are then useful probes to check self similarity. Wind tunnel data show indeed that

$S_{n}(\ell) \propto \ell^{\xi^{f}(n)}$,

where the set of values $\xi^{f}(n)$, usually called scaling exponents, is a nonlinear function of $n$. This result shows that the probability distribution functions of normalized velocity fluctuations do not collapse on the same shape at different scale, the shape varying from a gaussian at large scale to functions which display more and more important tails as the scale decreases (Frisch, 1995).

This behavior has been interpreted as the signature of the fact that the turbulent nonlinear energy cascade is not a self similar (fractal) but rather a multifractal process, characterized by the presence of energy transfer rates which are different in different spatial positions. In particular it means that at small scales fluctuations much larger than their rms value can be found in some particular spatial position. This phenomenon, known as spatial intermittency, was taken into account in his phenomenology two decades later by Kolmogorov (1962), who supposed that $\xi^{f}(n)=n / 3+\tau^{f}(n / 3)$. In ordinary fluids the correction due to intermittency is rather small on low order moments $(\tau(2 / 3) \simeq 0.02)$; one has to go up to the sixth order to obtain the "intermittency parameter" $\tau^{f}(3) \simeq 0.3-0.4$.

The MHD case is better analyzed in terms of the of the Elsässer (1950) variables $z^{\sigma}$ defined by $z^{\sigma}=\boldsymbol{v}+$ $\sigma \boldsymbol{B} /(\sqrt{4 \pi \rho})$ with $\sigma= \pm 1$. The equations governing incompressible MHD are then written

$\frac{\partial z^{\sigma}}{\partial t}+\left(z^{-\sigma} \cdot \nabla\right) z^{\sigma}=-\frac{1}{\rho} \nabla\left(p+\frac{B^{2}}{8 \pi}\right)+$ diss. terms.

The nonlinear structure of these equations is much the same as that of Navier Stokes equations so that we can expect a Kolmogorov-like scaling for the rms of field difference $\delta z_{\ell}^{\sigma}=z^{\sigma}(r+\ell)-z^{\sigma}(r)$

$<\left(\delta z_{\ell}^{\sigma}\right)^{2}>^{1 / 2} \propto \ell^{\frac{1}{3}}$.

Actually in MHD a different physical effect could be at work (Kraichnan, 1965; Dobrowolny et al., 1980): nonlinear interactions take place between eddies of different $\sigma$. Such eddies propagate in opposite directions at the Alfvén velocity corresponding to large scale magnetic field. This effect reduces the efficiency of the nonlinear cascade, as a consequence the Kraichnan's scaling is recovered

$$
<\left(\delta z_{\ell}^{\sigma}\right)^{2}>^{1 / 2} \propto \ell^{\frac{1}{4}} .
$$

When calculating structure functions for solar wind MHD fluctuations following Kolmogorov (1962) we can expect that

$S_{n}(\ell) \propto \ell^{\xi \mathrm{mhd}(n)}$ 


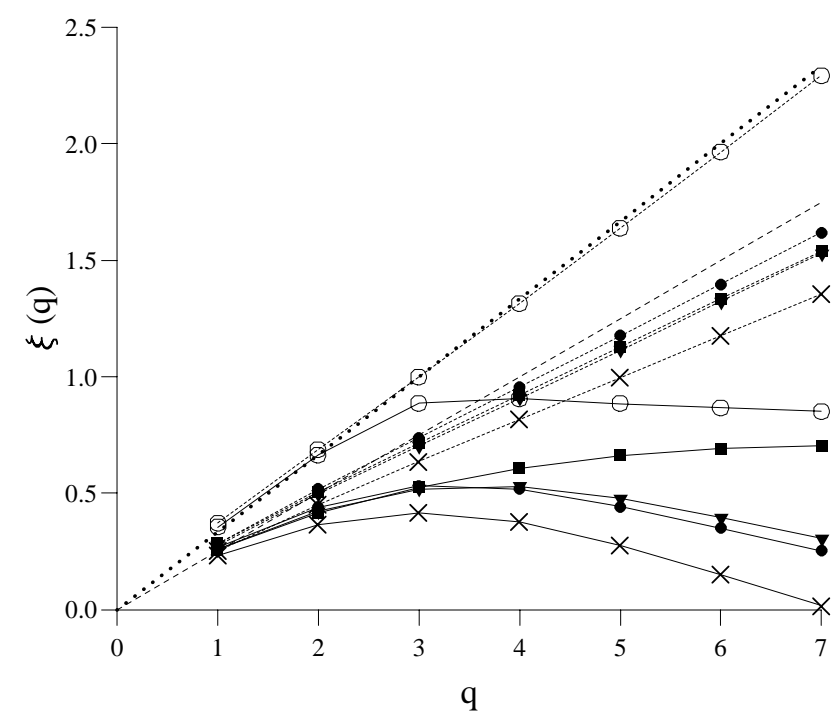

Fig. 1. Scaling exponents of the unconditioned (full lines) and conditioned (dashed lines) structure functions are shown as function of the order for different fluid velocities ( $V_{x}$ open circles, $V_{y}$ full squares) and magnetic field ( $B_{x}$ full triangles, $B_{y}$ full circles, $B_{z}$ crosses) components. Kolmogorov (dotted line) and IroshnikovKraichnan (long-dashed) scaling are also reported for comparison.

with either

$\xi^{\mathrm{mhd}}(n)=\frac{q}{3}+\tau^{\mathrm{mhd}}\left(\frac{n}{3}\right)$

for Kolmogorov's scaling or

$\xi^{\mathrm{mhd}}(n)=\frac{q}{4}+\tau^{\mathrm{mhd}}\left(\frac{n}{4}\right)$

for Kraichnan's scaling.

A number of structure function analysis performed both on solar wind and on laboratory plasma data have shown that both velocity and magnetic field fluctuations are indeed intermittent, i.e. $\xi^{\text {mhd }}(n)$ is a nonlinear as function of $n$ (Marsch and $\mathrm{Tu}, 1997)$ and that probability distribution functions of fluctuations are non gaussian (Sorriso-Valvo et al., 1999). Moreover the use of wavelet analysis described extensively in Veltri et al. (1999), has allowed to identify the nature of the coherent intermittent structures, which are responsible for the deviation with respect to self similarity.

\subsection{Haar wavelet structure functions analysis}

An alternative way to calculate structure functions, is based on the differencing characteristics of Haar wavelet (Mahrt, 1991; Katul et al., 1994; Veltri et al., 1999).

The wavelet trasform of a real square integrable signal $f(x)$ is defined as

$W_{f}(b, a)=C_{g}^{-1 / 2} \frac{1}{\sqrt{a}} \int_{-\infty}^{\infty} \psi\left(\frac{x-b}{a}\right) f(x) d x$,

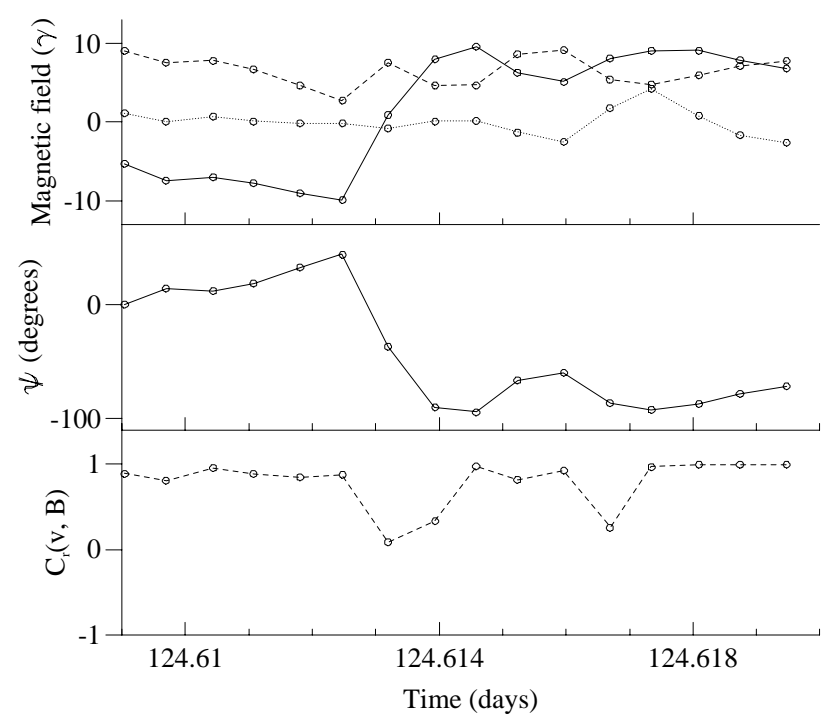

Fig. 2. A current sheet intermittent event: the three components of the magnetic field obtained through a minimum variance analysis (upper panel); the angle of rotation of the magnetic field in the plane perpendicular to the minimum variance direction (medium panel); the coefficient of correlation between velocity and magnetic field fluctuations (lower panel).

where $a$ is a scale dilation and $b$ a position traslation. The Haar basis is given by

$\psi(x)= \begin{cases}1 & \text { for } 0<x<1 / 2 \\ -1 & \text { for } 1 / 2 \leq x<1 \\ 0 & \text { otherwise }\end{cases}$

A logarithmically uniform spacing for scale discretization can be used, with increasingly coarser spatial resolution at larger scale. A complete orthogonal wavelet basis requires the same number $N$ of wavelet coefficients as the number of measurements, while for the scale dilation and for the position traslation the discretizations $a=2^{m}$ and and $b=2^{m} i$ are usually assumed, with $m$ and $i$ integer numbers such that $1 \leq m \leq M=\log _{2}(N)$ and $1 \leq i \leq 2^{M-m}$. The wavelet trasform of a given flow variable $\phi(x)$ can then be represented as function of $i$ and $m$, say $W_{\Phi}(i, m)$.

Haar wavelets can be used to define for any flow variable a set of functions, which are not strictly structure functions, as they are usually defined, but have the same physical meaning:

$$
<|\Phi(r+\ell)-\Phi(r)|^{q}>\sim \frac{<\left|W_{\Phi}(i, m)\right|^{q}>}{\left(2^{m / 2} \Delta\right)^{q}},
$$

where $\ell=2^{m} \Delta$ is the separation distance, $r=2^{m} i \Delta$ is the position and $\Delta$ is the data sampling length. The functions calculated according to Eq. (13) should have the same scaling laws as the structure functions, so in the following we will refer to them as structure functions without any further specification. From the structure functions the scaling exponents $\xi(q)$ can be calculated through a best fit of Eq. (8) over the interval of scale lengths $2 \Delta<\ell<2^{M} \Delta$. 


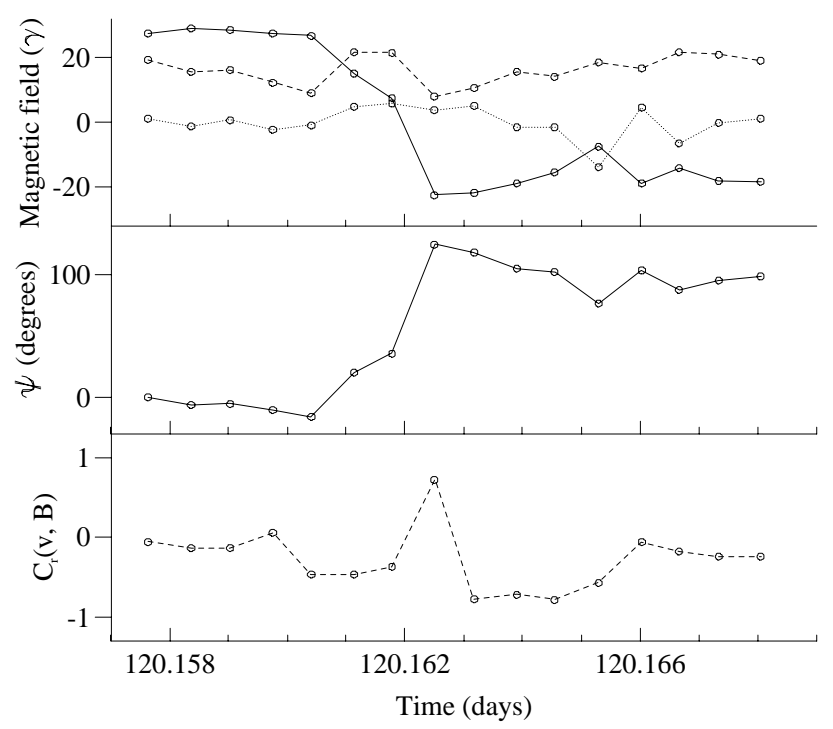

Fig. 3. A current sheet intermittent event: the three components of the magnetic field obtained through a minimum variance analysis (upper panel); the angle of rotation of the magnetic field in the plane perpendicular to the minimum variance direction (medium panel); the coefficient of correlation between velocity and magnetic field fluctuations (lower panel).

This technique has been applied by Veltri et al. (1999) to fluid velocity and magnetic field measurements performed during about 1 year in the space experiment ISEE. In this experiment only two components of the fluid velocity, namely, $V_{x}$ and $V_{y}$, have been obtained, together with all three components of the magnetic field. The reference frame used was the standard GSE frame and the sample was formed by data at a time resolution of $T=1 \mathrm{~min}$, so that the sampling rate was $\Delta=V_{s w} T \sim 24000 \mathrm{~km}$ ( $V_{s w}$ is the average solar wind velocity). The results are given in Fig. 1 (the expected Kolmogorov and Kraichnan self similar scaling are also reported for comparison). Looking at this figure it is seen that intermittency strongly affects the scaling exponents of all the flow variables: their values deviate more and more from the expected linear relations with increasing the order of the structure function.

Following Katul et al. (1994), Veltri et al. (1999) have then introduced "conditioned structure functions". The definition of such functions is based on the idea that large isolated values of $W_{\Phi}(i, m)$ represent a signature of intermittency. The wavelet coefficients are then classified as "passive" if $\left|W_{\Phi}(i, m)\right|^{2}<F\left\langle\left|W_{\Phi}(i, m)\right|^{2}\right\rangle$ or "intermittent", when $\left|W_{\Phi}(i, m)\right|^{2}>F\left\langle\left|W_{\Phi}(i, m)\right|^{2}\right\rangle$; structure functions are finally calculated by eliminating from the average in Eq. (13) the intermittent points. Note that $F$ was chosen in such a way to eliminate intermittency effects from the scaling exponents of the "conditioned structure functions". The results obtained were largely independent from the exact value of $F$. Actually, the "conditioned structure functions" were calculated for $F=5$. Less than 5\% of wavelets coefficients were eliminated from the average.

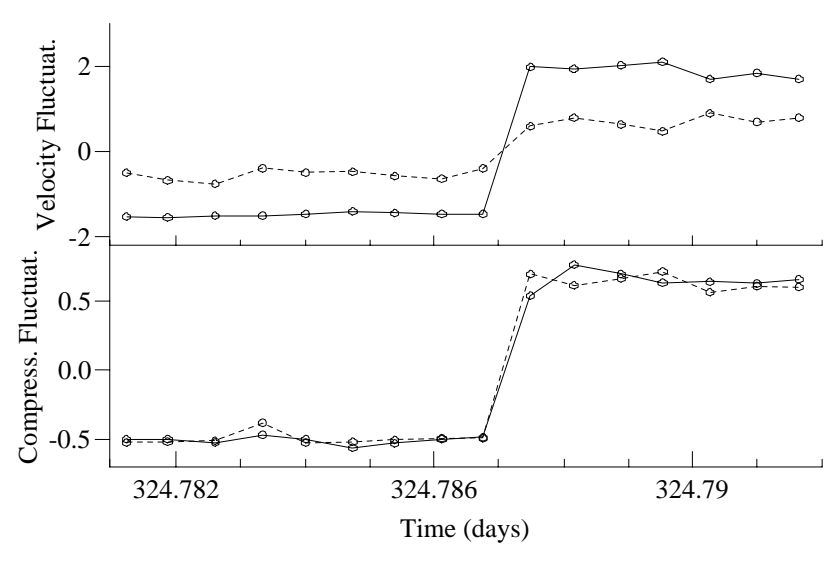

Fig. 4. A parallel shock intermittent event: $V_{x}$ (full line) and $V_{y}$ (dashed line) fluctuations normalised to the local average sound velocity (upper panel); proton density (full line) and sound velocity (dashed line) fluctuations normalised to their local average values (lower panel).

In Fig. 1 are represented also the scaling exponents derived from "conditioned structure functions". It can be seen that the relation $\xi(q)$ is almost linear for all flow variables, confirming that deviations from self similarity are effectively due to intermittent points identified through the corresponding large values of $W_{\Phi}(i, m)$.

\subsection{Nature of intermittency in solar wind}

The classification of wavelet coefficiens performed above, allows for an identification and a study of the most intermittent events in solar wind turbulence, which occur in those positions where the amplitude of the wavelet trasform displays the largest values compared to the average. These events, which occur on time scale of the order of few minutes, exhibit a small number of typical profiles, summarized as follows:

a) "tangential discontinuities": these structures are almost incompressible, $\left(\delta \rho \sim \delta T_{p} \sim 0\right)$ pressure balanced one dimensional current sheets. A minimum variance analysis performed on the magnetic field around the singularity shows (Figs. 2 and 3) that the component of the magnetic field which varies most changes sign, and this component is perpendicular to the average magnetic field (the magnetic field component along the third axis being almost zero). The magnetic field rotates then in a plane by an angle which is about $120^{\circ}-130^{\circ}$. There is one more interesting property: when these structures occur during an Alvénic period (velocity and magnetic field fluctuations highly correlated), the Alfvénic correlations goes from 1 to zero during the traversal of the current sheet (Fig. 2), when, on the contrary, these structures occur during a period of almost no Alfvénic correlation, the correlation increases to about 1 at the current sheet location (Fig. 3). 


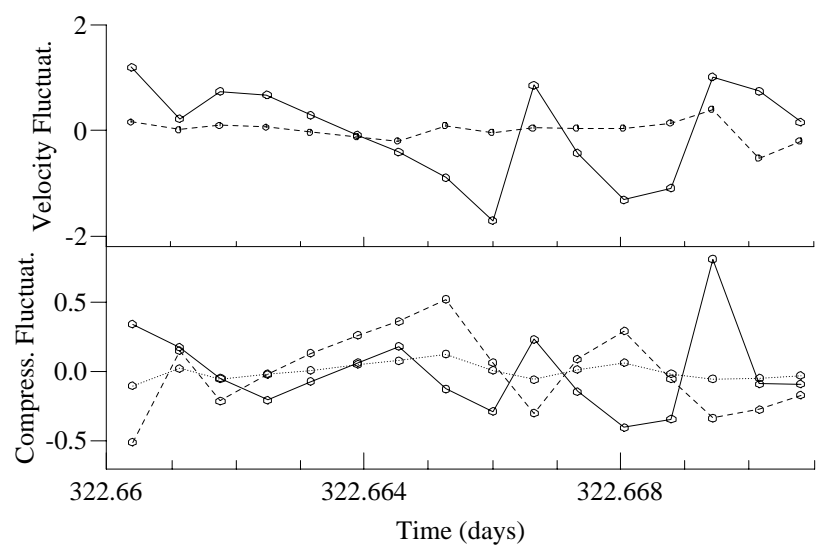

Fig. 5. A slow shock intermittent event: velocity fluctuations parallel (full line) and perpendicular (dashed line) to the local average magnetic field normalised to the local average sound velocity (upper panel); proton density (full line), sound velocity (dashed line) and total pressure (dotted line) fluctuations normalised to their local average values (lower panel).

b) "compressive discontinuities": these structures can be either parallel shocks, mainly observed on the radial component of the velocity field, but clearly seen also on the magnetic field intensity, proton temperature and density (Fig. 4); or slow mode wavetrains, characterized by a value of $\beta \sim 0$, a constant pressure, anticorrelated density and proton temperature fluctuations and with velocity fluctuations along the average magnetic field (Fig. 5).

All these structures are 1D, at variance with those observed in fluid turbulence (She et al., 1990). This result represent a direct confirmation of the conjecture by Carbone et al. (1995) about the topology of the most intermittent structures in solar wind. By comparing scaling exponents in fluid experiments with those in solar wind data, these authors have found that the former were consistent with model of intermittency (She and Leveque, 11994; Grauer et al., 1994; Politano and Pouquet, 1995) where the most intermittent structures had codimension 1 , while the latter require intermittent structures of codimension 2 .

\section{Coronal nanoflares modelling}

The results obtained when analyzing intermittent structures in solar wind MHD turbulence, described in the previous section, can furnish a key in modifying Parker's conjecture about smallest $\left(\sim 10^{24} \mathrm{erg}\right)$ flares occurrence. Actually Parker (1988) conjectured that these flares, which he called "nanoflares", should "correspond to dissipation of many small current sheets, forming in the bipolar regions as a consequence of the continous shuffling and intermixing of the footpoints of the field in the photospheric convection". These current sheets are nothing but "tangential discontinuity which become increasingly severe with the continuing wind-

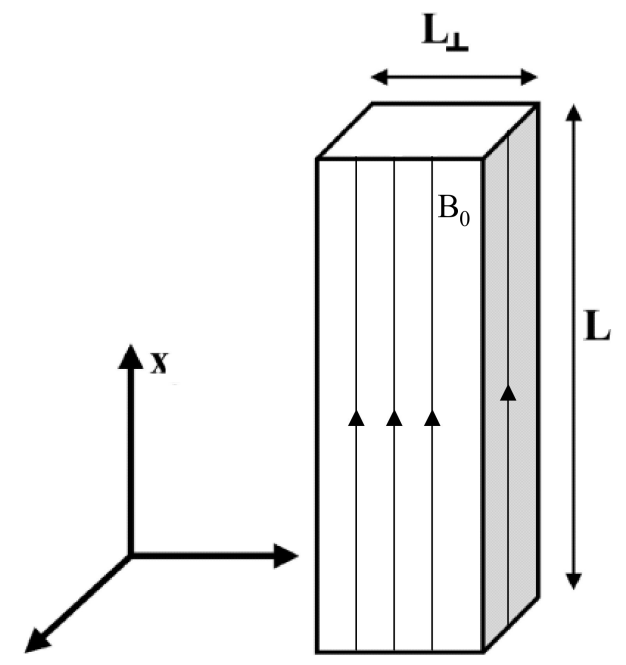

Fig. 6. A rough sketch of the loop model.

ing and interweaving eventually producing intense magnetic dissipation in association with magnetic reconnection".

It is now worth tempting to suppose that "nanoflares" correspond to dissipation of many small current sheets, "forming in the nonlinear cascade" occuring inside coronal magnetic structures as consequence of the power input due to footpoint motion. Current sheets are nothing but "coherent intermittent small scale structures" of MHD turbulence. Energy injection at large scales would be essentially due to photospheric motions; nonlinear effects would be responsible for energy transfer to small scales, while the intermittent nature of energy releases would be the result of a "turbulent energy cascade", from large spatial/temporal scales down to small dissipative scales. Finally, some form of dissipation would turn the turbulence energy into thermal or other forms of energy.

To investigate the consequences of this conjecture, we need to build up a model to describe MHD turbulence evolution inside a coronal loop.

\subsection{The RMHD shell model for turbulence in coronal loop}

In a simplified form a loop can be represented as box, with a large aspect ratio $R=L / L_{\perp} \gg 1$ ( $L$ being the height and $L_{\perp}$ the side of its square basis), inside which there is a region of uniform density $\rho$ and uniform magnetic field $B_{0}$, whose direction is vertical (Fig. 6).

A coronal magnetic loop have $\beta \sim 10^{-2}$ ( $\beta$ being the kinetic to magnetic pressure ratio) and a small perpendicular to longitudinal magnetic field ratio $B_{\perp} / B_{0}<1 / R$. Starting from MHD Eqs. (5) and using the above assumptions, a set of RMHD equations (Strauss, 1976; Zank and Matthaeus, 1992) can be obtained, which ensures that the plasma behaves as an incompressible fluid in the directions perpendicular to $\boldsymbol{B}_{0}$, while only propagation at the Alfvén speed takes place in the 
direction parallel to $\boldsymbol{B}_{0}$ :

$$
\begin{gathered}
\frac{\partial \boldsymbol{Z}^{\sigma}}{\partial t}+\left(\boldsymbol{Z}^{-\sigma} \cdot \nabla_{\perp}\right) \boldsymbol{Z}^{\sigma}+\nabla_{\perp} p-\sigma \frac{\partial \boldsymbol{Z}^{\sigma}}{\partial x}=\chi \nabla_{\perp}^{2} \boldsymbol{Z}^{\sigma} \\
\nabla_{\perp} \cdot \boldsymbol{Z}^{\sigma}=0,
\end{gathered}
$$

where $\boldsymbol{Z}^{\sigma}=\boldsymbol{v}_{\perp}+\sigma \boldsymbol{b}_{\perp}$ (with $\sigma= \pm 1$ ) are the Elsässer variables; $\boldsymbol{v}_{\perp}, \boldsymbol{b}_{\perp}$ and $\nabla_{\perp}$ are the component perpendicular to $\boldsymbol{B}_{0}$ of the velocity, magnetic field and gradient, respectively; $p$ is the total (kinetic + magnetic) pressure; $x$ is the coordinate along the loop. Lenghts are normalized to the loop length $L$, velocities to the Alfvén velocity $c_{A}=\boldsymbol{B}_{0} / \sqrt{4 \pi \rho}$ ( $\rho$ is the mass density); time is normalized to the Alfvén transit time $T_{A}=L / c_{A}$ (the time an Alfvén wave takes to propagate along the loop); the magnetic field is normalized to $\boldsymbol{B}_{0}$ and the pressure is normalized to $\rho c_{A}^{2}$. The dissipation coefficient is $\chi=\mu /\left(c_{A} L\right)$, where the the magnetic diffusivity $\mu$ has been assumed equal to the kinematic viscosity $\nu$. Condition (15) ensures that the plasma behaves as an incompressible fluid in the directions perpendicular to $\boldsymbol{B}_{0}$, while only propagation at the Alfvén speed takes place parallel to $\boldsymbol{B}_{0}$.

Direct simulations (Einaudi et al., 1996; Hendrix and Van Hoven, 1996; Dmitruk and Gomez, 1997, 1999; Dmitruk et al., 1998) of 2D and 3D MHD equations, and within the framework of RMHD have been performed, where the connection between dissipation bursts in turbulence and nanoflares has been introduced. However, simulation are subject to a severe limitation: the very high Reynolds/Lundquist numbers typical of the coronal plasma are not accessible. This does not allow to describe the transition from the fractal to the multifractal behaviour which is associated with intermittency in turbulent flows. In this respect, MHD shell models represent a useful tool, since they allow to model high Reynolds/Lundquist number turbulence with a small computational effort. Boffetta et al. (1999) have applied a MHD shell model to study the statistical properties of dissipated energy in the coronal turbulence. However, the application of shell models to the turbulence in coronal plasmas suffers for two main limitations:

a) MHD shell models are derived within the incompressible MHD (Boffetta et al., 1999), corresponding to the limit $\beta \gg 1$ ( $\beta$ being the kinetic to magnetic pressure ratio). On the contrary, the magnetically dominated coronal plasma $\left(\beta \sim 10^{-2}\right)$ is better represented by reduced MHD: in such an approach only noncompressive fluctuations polarized perpendicular to the main magnetic field $\boldsymbol{B}_{0}$ are retained, while nonlinear coupling take place only in the directions orthogonal to $\boldsymbol{B}_{0}$.

b) shell models do not give any information neither on the spatial structure of the turbulence nor on the geometry of the considered physical system. For instance, the energy input from photospheric motions is represented by a forcing term acting on the large-scale shells, but delocalized in space.

To overcome the above drawbacks we have built up (Nigro et al., 2004) a new kind of shell model. This model is derived in the framework of the above written RMHD (Eq. 14), instead of incompressible MHD. Dynamical variables depend on the coordinate $x$ parallel to the main magnetic field $\boldsymbol{B}_{0}$ in order to preserve the spatial information along this direction. We will refer to this model as "hybrid shell model".

From Eq. (14) the equations for the "hybrid shell model" can be obtained, where a Fourier transform of the two coordinates perpendicular to $\boldsymbol{B}_{0}$ is performed, while the dependence on space variable $x$ along $\boldsymbol{B}_{0}$ is kept:

$$
\begin{aligned}
& \left(\frac{\partial}{\partial t}-\sigma \frac{\partial}{\partial x}\right) Z_{n}^{\sigma}(x, t)=-\chi k_{n}^{2} Z_{n}^{\sigma}(x, t)+ \\
& \mathrm{i} k_{n}\left(\frac{11}{24} Z_{n+1}^{\sigma} Z_{n+2}^{-\sigma}+\frac{13}{24} Z_{n+1}^{-\sigma} Z_{n+2}^{\sigma}-\frac{19}{48} Z_{n+1}^{\sigma} Z_{n-1}^{-\sigma}-\right. \\
& \left.\frac{11}{48} Z_{n+1}^{-\sigma} Z_{n-1}^{\sigma}+\frac{19}{96} Z_{n-1}^{\sigma} Z_{n-2}^{-\sigma}-\frac{13}{96} Z_{n-1}^{-\sigma} Z_{n-2}^{\sigma}\right)^{*}
\end{aligned}
$$

with $n=0,1, \ldots, n_{\max }$ and $\sigma= \pm 1$. The model is built up by following a standard procedure: the $\boldsymbol{k}$-space perpendicular to $\boldsymbol{B}_{0}$ is divided into concentric shells of exponentially growing radius; for each shell a scalar value $k_{n}=k_{0} 2^{n}$ of the wavevector and a scalar value $Z_{n}^{\sigma}(x, t)$ of the Elsässer variables is defined (the fundamental dimensionless wavevector is $\left.k_{0}=2 \pi\left(L / L_{\perp}\right)=2 \pi R\right)$. The evolution equations for the dynamical variables $Z_{n}^{\sigma}(x, t)$ are built up by retaining only the interactions between nearest and next nearest neighbor shells in the form of quadratic nonlinearities. The nonlinear terms coefficients are then determined by imposing that they conserve the 2D quadratic invariants: total energy, cross helicity and squared magnetic potential (Giuliani and Carbone, 1998).

\subsection{Boundary conditions}

According to Eq. (16), the quantities $Z_{n}^{+}$propagate in the negative $x$ direction at the Alfvén speed, while $Z_{n}^{-}$propagate in the positive $x$ direction. At the lower boundary $x=0$ we will then only impose the value of $Z_{n}^{-}$, while at the upper boundary $x=1$ we will impose the value of $Z_{n}^{+}$. Using the relation between $Z_{n}^{\sigma}$ and velocity field harmonics $v_{\perp n}(x, t)=\left(Z_{n}^{+}(x, t)+Z_{n}^{-}(x, t)\right) / 2$ the expression for the boundary values of the "entering" dynamical variables can be obtained

$$
\begin{gathered}
Z_{n}^{-}(x=0, t)=2 v_{\perp n}(x=0, t)-Z_{n}^{+}(x=0, t), \\
Z_{n}^{+}(x=1, t)=2 v_{\perp n}(x=1, t)-Z_{n}^{-}(x=1, t) .
\end{gathered}
$$

On the RHS of these equations the velocity $v_{\perp n}$ must be specified, while the Elsässer variables, corresponding to perturbations leaving the domain $\left(Z_{n}^{+}(x=0, t)\right.$ and $\left.Z_{n}^{-}(x=L, t)\right)$, are determined by the evolution Eqs. (16) inside the domain. We have chosen to inject energy only through the lower boundary, keeping a vanishing velocity at the upper boundary: $v_{\perp n}(x=1, t)=0$. Condition (18) then implies $Z_{n}^{+}=-Z_{n}^{-}$at $x=1$; i.e. exiting perturbations are totally reflected at the upper boundary. This boundary condition has been chosen for the sake of simplifying the model; 
however, we expect that the results would remain essentially unchanged if energy exchanges were be allowed also through the upper boundary. Moreover, we have assumed that most of energy in photospheric motions is concentrated at larger spatial scales $\sim L_{\perp}$. Then, at the lower boundary $x=0$ only the first three shell, which represent the minimum number of shells which must be populated in order to initiate nonlinear interactions, are excited:

$v_{\perp n}(x=0, t)=u_{\perp} f_{n}(t), n=0,1,2$,

$v_{\perp n}(x=0, t)=0, n=3, \ldots, n_{\max }$.

The constant $u_{\perp}$ gives the order of magnitude of velocity perturbations at the boundary, while $f_{n}(t)$ are random signals gaussian distributed, with unit standard deviation, selfcorrelated with a correlation time $t_{c}$, which represents the characteristic time associated with photospheric motions. We used $t_{c}=300 \mathrm{~s}$. From Eq. (16) an energy balance equation can be derived

$\frac{d E}{d t}=F(t)-W(t)$,

where $E(t), F(t)$ and $W(t)$ represent, respectively, the total energy, the net incoming energy flux and the total dissipated power

$E=\sum_{\sigma} \int_{0}^{1} \varepsilon^{\sigma}(x, t) d x, \quad W=\frac{\chi}{2} \sum_{\sigma, n} \int_{0}^{1} k_{n}^{2}\left|Z_{n}^{\sigma}(x, t)\right|^{2} d x$,

$F=\sum_{\sigma}\left[F^{\sigma}(x=0, t)-F^{\sigma}(x=1, t)\right]$,

where $\varepsilon^{\sigma}(x, t)$ and $F^{\sigma}(x, t)$ are the pseudoenergy per height unit and the pseudoenergy flux

$\varepsilon^{\sigma}(x, t)=\frac{1}{4} \sum_{n=0}^{n_{\max }}\left|Z_{n}^{\sigma}(x, t)\right|^{2}, \quad F^{\sigma}(x, t)=-\sigma \varepsilon^{\sigma}(x, t)$.

Equation (19) has also been used to check the accuracy of the numerical solution.

\section{Numerical results}

Equations (16) have been numerically solved, using a second order finite difference scheme in space and a second order Runge-Kutta method in time.

As a typical case we chosen to study a magnetic structure with a parallel length $L=3 \times 10^{4} \mathrm{~km}$, and an aspect ratio $R=\left(L / L_{\perp}\right)=30 / 2 \pi$, a characteristic Alfvén velocity $c_{A}=2 \times 10^{3} \mathrm{~km} \mathrm{~s}^{-1}$ (corresponding to Alfvén transit time $T_{A}=15 \mathrm{~s}$ ). The velocity at the forcing boundary has been assumed to be of the order of $1 \mathrm{~km} \mathrm{~s}^{-1}$ corresponding to that observed in photospheric motions, so that $u_{\perp}=5 \times 10^{-4}$; finally a very small dissipation coefficient $\chi=10^{-7}$ has been used, which requires $n_{\max }=11$ in order to ensure that the generated spectrum will be fully contained into the finite number $n_{\max }$ of shells. Using the above values and a typical mass density $\rho=1.67 \times 10^{-16} \mathrm{~g} \mathrm{~cm}^{-3}$, the following normalization factors for energy and power are obtained $E_{N}=\left(\rho c_{A}^{2} L^{3}\right) / R^{2}=7.93 \times 10^{27} \mathrm{erg}, W_{N}=$

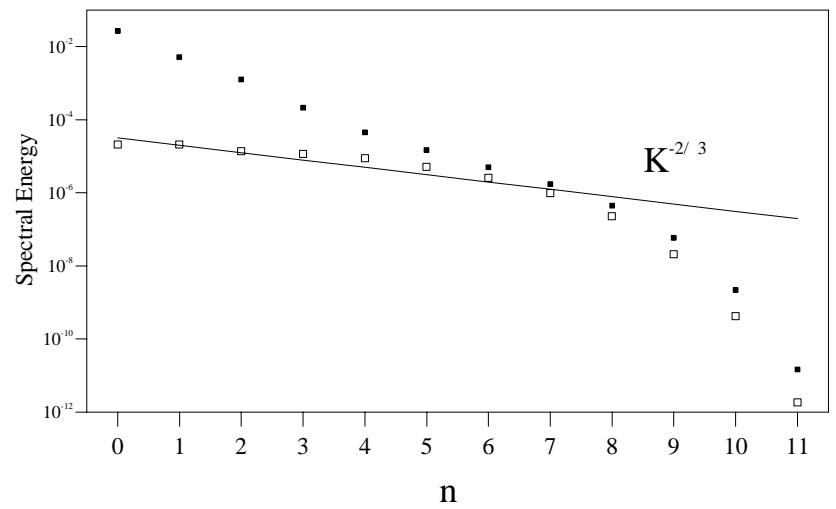

Fig. 7. Kinetic (white boxes) and magnetic (black boxes) spectral energy as function of the shell order. The spectra are averaged both in direction along the loop and in time.

$\left(\rho c_{A}^{3} L^{2}\right) / R^{2}=5.28 \times 10^{26} \mathrm{erg} \mathrm{s}^{-1}$. Note that $E_{N}$ is also an estimation of the d.c. magnetic energy stored in the loop.

\subsection{Energetics}

As described above, energy is injected in the first three shells at the lower boundary. In consequence of propagation parallel to $\boldsymbol{B}_{0}$, this energy is distributed along the $x$ direction. At the same time, nonlinear couplings transfer this energy to smaller scales in the transverse direction. In this way a spectrum is formed (Fig. 7), ranging from the large injection scales $(n \leq 2)$ down to the small dissipative scales $(n \geq 9)$. In the inertial domain $(3 \leq n \leq 8)$ the characteristic Kolmogorov power law spectrum is displayed mainly by kinetic energy.

The time evolution for total energy, net incoming energy flux and dissipated power is shown in Fig. 8. At the initial time the magnetic structure does not contain energy. During a transient, which lasts about $15 \mathrm{~h}$, the energy increases: the motions at the base of the loop inject Alfvénic perturbations which gradually fill the whole structure. With increasing time, the energy does not stabilize but displays strong irregular variations. These variations are due both to energy input/output at the lower boundary (the upper is a perfectly reflecting boundary), and to dissipation. The energy input is not simply determined by the external forcing, but it depends also on how the forcing couples with perturbations which are present inside the system. In particular, this coupling contributes to determine the sign of the net energy flux. In Fig. 8 it can be seen that the energy flux continuously changes sign on a short time scale.

Dissipation gives a negative definite contribution to energy variations. The time dependence of the dissipated power shown in Fig. 8 indicates that dissipation takes place in an intermittent fashion: periods characterized by the presence of "strong dissipation peaks" are observed, along with relatively "quiet" periods, during which the level of dissipation is much lower. The strongest event correspond to a peak dissipated power of $\sim 10^{24} \mathrm{erg} \mathrm{s}^{-1}$. We expect that further events 


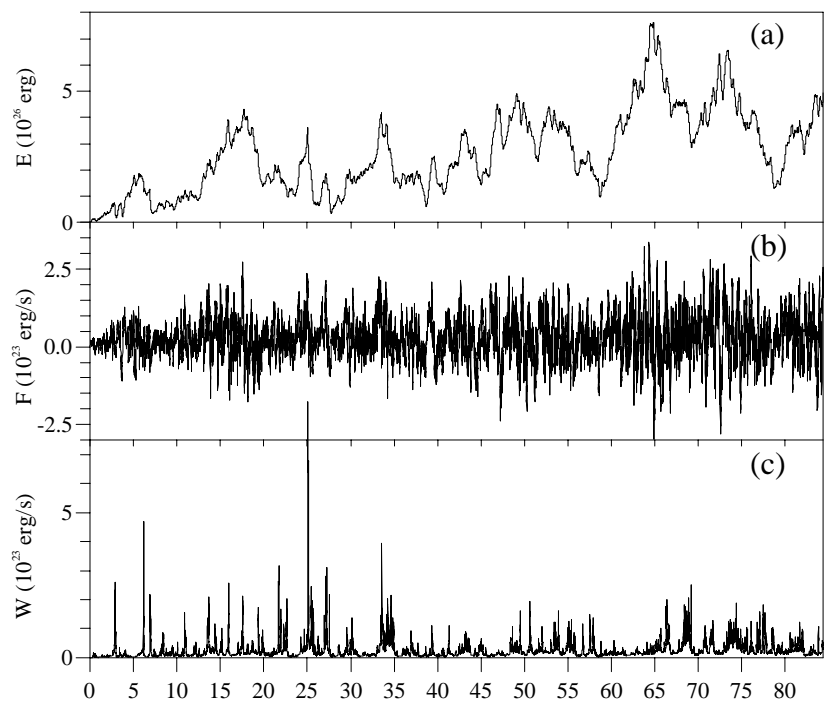

Fig. 8. Time evolution of (a) Energy, (b) Energy flux and (c) Dissipated power.

which such a strong dissipated power would be observed in longer simulations. Even though the energy never reaches a stationary state, when observed over very long times it oscillates around an average value. This indicates that a statistical equilibrium is reached between incoming flux, outcoming flux and dissipation. The energy and the dissipated power, averaged over a time which excludes the initial transient are $\langle E\rangle_{t} \simeq 3.84 \times 10^{-2}$ and $\langle W\rangle_{t} \simeq 5.05 \times 10^{-5}$. In a similar way, we calculated the average incoming and outcoming fluxes, by separately considering periods of positive $\left\langle F_{\text {in }}\right\rangle_{t} \simeq 8.38 \times 10^{-5}$ and periods of negative flux $\left\langle F_{\text {out }}\right\rangle_{t} \simeq-2.99 \times 10^{-5}$. Then about $60.3 \%$ of the energy which enter the system during the whole simulation is dissipated, while $35.7 \%$ propagate outside. Average fluxes and dissipation tend to cancel out, a small unbalance remaining $\langle d E / d t\rangle_{t} /\left\langle F_{\text {in }}\right\rangle_{t} \simeq 4 \%$ which tends to vanish increasing the simulation time.

The average energy can be splitted as the sum of the kinetic $\left\langle E_{K}\right\rangle_{t}=8.51 \times 10^{-5}$ and magnetic $\left\langle E_{M}\right\rangle_{t}=$ $3.84 \times 10^{-2}$ average energies. From the average energy an estimation of the perturbation amplitude can be obtained $\delta B_{\perp} / B_{0} \sim\left\langle E_{M}\right\rangle_{t}^{1 / 2} \simeq 0.2$, and $\delta v_{\perp} / c_{A} \sim\left\langle E_{K}\right\rangle_{t}^{1 / 2} \simeq$ $9.2 \times 10^{-3}$. This means that magnetic field fluctuations inside the loop dominate over velocity fluctuations at least at larger scales. This result is a consequence of the fact that the forcing at the loop base has a characteristic time $t_{c}$ much larger than the Alfvén transit time $T_{A}$ (Einaudi and Velli, 1999). In particular, this implies that the reduced cross-helicity of fluctuations $H=2 \delta v \delta b /\left(\delta v^{2}+\delta b^{2}\right) \simeq 2 \delta v / \delta b \sim 10^{-1}$. This condition is equivalent to $\delta Z^{+} \simeq \delta Z^{-}$and it corresponds to the presence of Alfvénic fluctuations propagating along the loop in both directions. This holds during the whole simulation, except for a short initial transient $\left(\sim 2 T_{A}\right)$. The condition $|H| \ll 1$ implies that nonlinear interactions are effective in transfering energy along the spectrum.
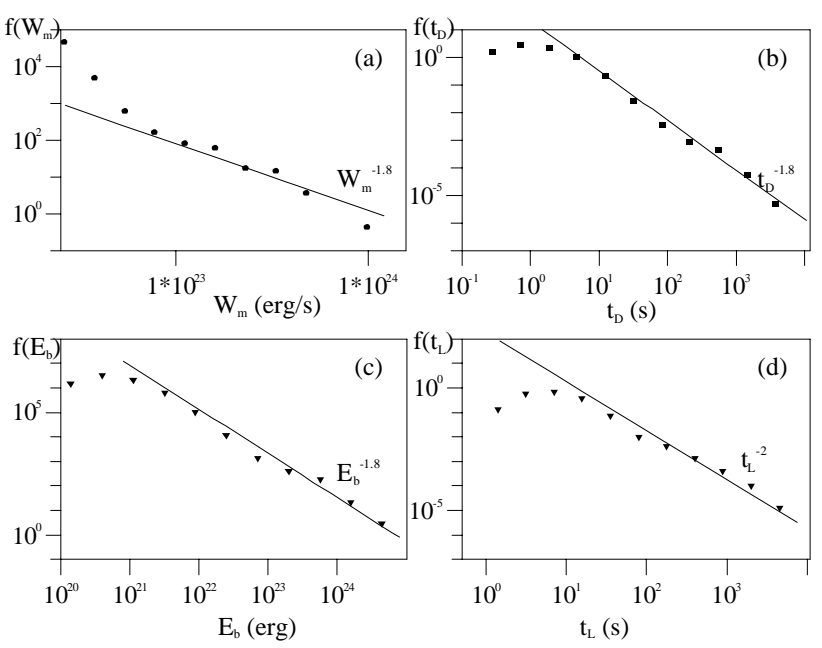

Fig. 9. Probability distribution functions for (a) Peak of dissipated power, (b) Duration, (c) Burst Energy, (d) Time separation. Power law with indices 1.8 for the first three distributions and 2 for the last one are represented for comparison.

The velocity fluctuations inside the loop are considerably larger with respect to photospheric motions which drive the energy input. This means that loop works as an extremely efficient energy storage device.

\subsection{Probability distribution functions}

The time dependence of dissipated power shown in Fig. 8 displays a sequence of spikes, which are supposed to represent the energy release events (micro or nanoflares) observed in the solar corona. We have then calculated the statistical properties of such events to compare them with the observed properties of coronal flares. So we have defined a burst of dissipation by the condition $W(t) \geq W_{\mathrm{thr}}$. We have chosen the threshold value as $W_{t h r}=W_{\text {ave }}+2 \sigma$, where the average and the standard deviation of the dissipated power have been calculated on the time intervals between bursts by an iterative process to take into account only the background contribution. The calculated PDFs of peak maximum power $f\left(W_{m}\right)$, peak duration time $f\left(t_{D}\right)$, energy dissipated in a burst $f\left(E_{b}\right)$ and waiting time between bursts $f\left(t_{L}\right)$ are represented in Fig. 9. All these distribution functions display power law behavior extended over a wide range of decades. The power law indices are of the order of 1.8 for the first three distribution and about 2 for the waiting times, values which are in agreement with those obtained in statistical analisys of impulsive events in the corona. The range of dissipated energy goes from $10^{20} \mathrm{erg}$ to $10^{25} \mathrm{erg}$, which compare very well with the energy involved in nanoflares. This wide dynamical range is due to intermittency in high Reynolds number turbulence, efficiently described by the shell technique. The energy of dissipation events depend on the value $E_{N}$ used to normalize energy. A loop containing a larger amount of magnetic energy in the equilibrium field would correspond to larger energies released in dissipative events. However, in 


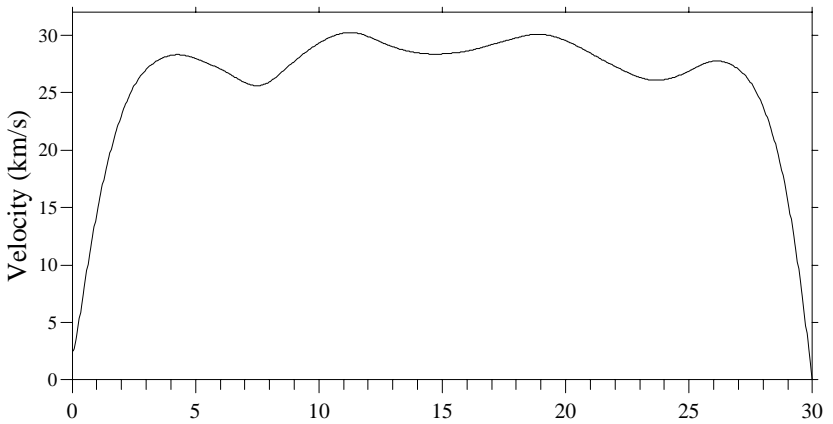

Fig. 10. Root mean square value of the velocity fluctuations as function of the coordinate along the loop.

our model there is an upper limit to the energy which can be released in a single event: this limit is represented by the energy of the equibrium field $\sim E_{N}$. This implies that our model cannot account for large flares, which probably need topologically complex magnetic field above active regions to store and dissipate the required energy.

\subsection{Velocity fluctuations}

A extremely efficient challenge for the model is represented by the comparison of velocity fluctuations obtained in the numerical model with unresolved non-thermal velocity. From the observational point of view the non-thermal velocity represents the excess amount of an observed line width over thermal contribution. The physical reason for the excess broadening is still not clear. In the framework of our model we can interpret the observed non-thermal velocity as turbulent velocity. In fact the isotropic and very small scale nature of non-thermal motion appears to be suited to MHD turbulence interpretation of non-thermal velocity.

The velocity distribution along the loop is represented in Fig. 10, where it can be seen that, starting from about $1 \mathrm{~km} / \mathrm{s}$ at the boundary, values of the order of $30 \mathrm{~km} / \mathrm{s}$ are obtained $3000 \mathrm{~km}$ higher. This distribution compares well with the distribution of unresolved velocity, observed by Sumer experiment (Chae et al., 1998), assuming that temperature is directly related to height above photosphere. The numerical values are also in agreement with observed coronal nonthermal velocity obtained by Brosius et al. (2000). In this work they have presented a high-resolution EUV spectrum of solar active region obtained with the SERTS sounding rocket. Using the strong emission line observed for each ionization stage of Fe from X though XVI and Ni XVIII, they find that all of the measured non-thermal line widths yield velocities consistent with $35 \mathrm{~km} / \mathrm{s}$, within the measurement uncertainties.

Even more interesting comparisons may be derived from the analysis of non-thermal velocity measured in correspondence of flares. Let us see what happens to velocity fluctuations in our numerical simulations. Looking at Fig. 11 it is seen that magnetic field fluctuations display variations on time scale of the order of several hours, much the same as

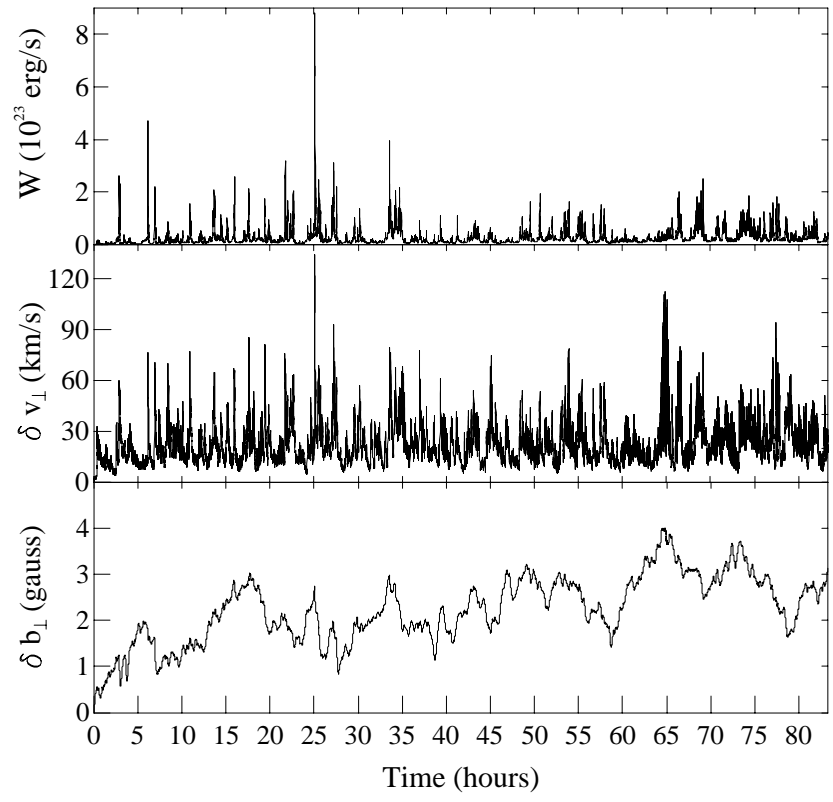

Fig. 11. Root mean square value of the velocity (middle panel) and magnetic field (lower panel) fluctuations as function of time. The dissipated power is represented in the upper panel for comparison.

the stored fluctuating energy (Fig. 8) of which they represent the main contribution. On the contrary, velocity fluctuations are characterized by spikes which can be as higher as $80-140 \mathrm{~km} / \mathrm{s}$, much larger then their average value $(\simeq 30$ $\mathrm{km} / \mathrm{s}$ ). Even if these spikes are not always correlated with a burst of dissipation, the contrary is always true: every dissipation burst is correlated with a spike in the velocity fluctuation. This behavior is clearly seen both in Fig. 11 and in Fig. 12 where a direct comparison between time behavior of dissipated power and velocity fluctuation amplitude is performed by zooming on a particular time period. The strong increase of velocity fluctuations in correspondence (just before) a dissipation burst compares extremely well with the increase of nonthermal velocity observed during flares, not only qualitatively but also quantitatively. In fact Landi et al. (2003) have observed maximum value of around $100 \mathrm{~km} / \mathrm{s}$ at flare onset and then decay to the values of around $30 \mathrm{~km} / \mathrm{s}$ non thermal mass motion of nonflaring plasma.

\section{Conclusions}

The results obtained strongly support the view that coronal nanoflares could be related to intermittent dissipative events in the MHD turbulence produced in a coronal magnetic structure by footpoint motions. The main physical ingredients in the model are represented by an energy injection at a boundary of the simulation domain, which is efficiently stored in the loop up to significant levels mainly in the form of magnetic fluctuations. Fast increase of fluctuating kinetic energy, allows to bring the accumulated magnetic energy from large scales to small scales. This transport is realized by nonlinear 


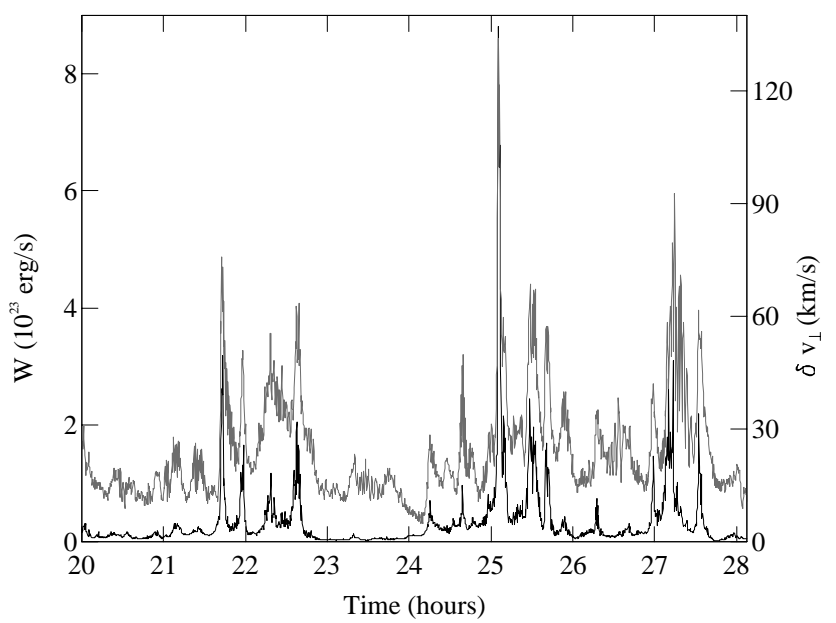

Fig. 12. Comparison between velocity fluctuations (grey line) and dissipated power (black line) time evolution.

interactions which process magnetic and kinetic fluctuations and give rise to the intermittent behavior, whose signature is found on statistical distribution of observational data, without any need of "ad hoc" hypothesis.

The "hybrid shell model" we have used is extremely useful in studying the energy balance of dynamical variables, but it does not allow for a description of the spatial characteristics of the turbulence. On the other hand, the data from solar wind have allowed us to understand what are the properties of the most intermittent structures in MHD turbulence, which as concerns incompressible MHD, turn out to be current sheets. Integrating the informations furnished by both methods represents then an extremely efficient way to look at problems which are far from the possibility of actual computers if Direct Numerical Simulations were used.

It is then worth summarizing some methodological results which follow from this paper:

a) physical understanding of complex nonlinear phenomena occurring in plasmas require the coordinated utilization of different tools: space and laboratory data analysis, simplified dynamical models, numerical simulations;

b) the signature of non linear interactions seems to be a multifractal intermittent behavior. This behavior observed in turbulent fluid flows seems to represent a key to explain some burstly phenomena occurring in space, laboratory and solar corona plasmas;

c) dynamical models represent a particularly efficient tool to describe this intermittent behavior.

Acknowledgements. The authors acknowledge useful discussion with P. Giuliani. This work was partially supported by the MIUR (Ministero dell'Istruzione, dell'Università e della Ricerca) through a National Project Fund (Cofin 2002) and by the European Community within the Research Training Network "Turbulence in Space Plamas, Theory, Observation and Simulation". Numerical calculations have been performed in the framework of HPCC (Center for High Performance Computing) of the University of Calabria.

Edited by: A. Chian

Reviewed by: two referees

\section{References}

Boffetta, G., Carbone, V., Giuliani, P., Veltri, P., and Vulpiani, A.: Power Laws in Solar Flares: Self-Organized Criticality or Turbulence?, Phys. Rev. Lett., 83, 22, 4662-4665, 1999.

Brosius, J. W. , Thomas, R. J., Davila, J. M., and Landi, E.: Analysis of a Solar Active Region Extreme-Ultraviolet Spectrum from SERTS-97, Astrophys. J., 543, 2, 1016-1026, 2000.

Carbone, V., Veltri, P., and Bruno, R.: Experimental Evidence for Differences in the Extended Self-Similarity Scaling Laws between Fluid and Magnetohydrodynamic Turbulent Flows, Phys Rev. Lett., 75, 17, 3110-3113, 1995.

Chae, J., Schühle, U., and Lemaire, P.: SUMER Measurements of Nonthermal Motions: Constraints on Coronal Heating Mechanisms, Astrophys. J., 505, 2, 957-973, 1998.

Crosby, N. B., Aschwanden, M. J., and Dennis, B. R.: Frequency distributions and correlations of solar X-ray flare parameters, Solar Phys., 143, 275-299, 1993.

Dmitruk, P. and Gomez., D. O.: Turbulent coronal heating and the distrinution of nanoflares, Astrophys. J., 484, L83-L86, 1997.

Dmitruk, P., Gomez., D. O., and De Luca, E. E.: Magnetohydrodynamic turbulence of coronal active regions and the distribution of nanoflares, Astrophys. J., 505, 974-983, 1998.

Dmitruk, P., and Gomez., D. O.: Scaling Law for the Heating of Solar Coronal Loops, Astrophys. J., 527, L63-L66, 1999.

Dobrowolny, M., Mangeney, A., and Veltri, P.: Fully developed anisotropic hydromagnetic turbulence in interplanetary space, Phys. Rev. Lett., 45, 144-147, 1980.

Einaudi, G., Velli, M., Politano, H., and Pouquet, A.: Energy Release in a Turbulent Corona, Astrophys. J., 457, L113-L116, 1996.

Einaudi, G. and Velli, M.: The distribution of flares, statistics of magnetohydrodynamic turbulence and coronal heating, Phys. Plasmas, 6, 11, 4146-4153, 1999.

Elsässer, W. M.: The Hydromagnetic Equations, Phys. Rev., 79, 183-183, 1950.

Fisch, U.: Turbulence, Cambridge University Press, Cambridge, UK, 1995.

Giuliani, P. and Carbone, V.: A note on shell models for MHD turbulence, Europhys. Lett., 43, 5, 527-532, 1998.

Grauer, R., Krug, J., and Marliani, C.: Scaling of high-order structure functions in magnetohydrodynamic turbulence, Phys. Lett. A, 195, 5-6, 335-338, 1994.

Hendrix, D. L., and Van Hoven, G.: Magnetohydrodynamic Turbulence and Implications for Solar Coronal Heating, 467, 887-893, 1996.

Katul, G. G., Albertson, J. D., Chu, C. R., and Parlange, M. B.: Intermittency in Atmosheric Surface Layer Turbulence: The Orthonormal Wavelet Representation, Wavelets in Geophysics, edited by Foufoula-Georgiou, E. and Kumar, P., Academic Press, 81-105, 1994.

Kolmogorov, A. N.: The local structure of turbulence in incompressible viscous fluid for very large Reynolds number, Dokl. Akad. Nauk. SSSR, 30, 299-303, 1941. 
Kolmogorov, A. N.: A refinement of previous hypotheses concerning the local structure of turbulence in a viscous incompressible fluid at high Reynolds number, J. Fluid Mech., 13, 82-85, 1962.

Kraichnan, R. H.: Inertial range spectrum in hydromagnetic turbulence, Phys. Fluids, 8, 1385-1387, 1965.

Landi, E., Feldman, U., Innes, D. E., and Curdt, W.: Mass Motions and Plasma Properties in the $107 \mathrm{~K}$ Flare Solar Corona, Astrophys. J., 582, 1, 506-519, 2003.

Lepreti, F., Carbone, V., and Veltri, P.: Solar Flare Waiting Time Distribution: Varying-Rate Poisson or Lévy Function?, Astrophys. J., 555, 2, L133-L136, 2001.

Lin, R. P., Schwartz, R. A., Kane, S. R., Pelling, R. M., and Hurley, K. C.: Solar hard X-ray microflares, Astrophys. J., 283, 421-425, 1984.

Mahrt, L.: Eddy Asymmetry in the Sheared Heated Boundary Layer, J. Atmos. Sci., 48, 3, 472-492, 1991.

Marsch E., and Tu, C. Y.: Intermittency, no-gaussian statistics and fractal scaling of MHD fluctuations in the Solar Wind, Nonlin. Proc. Geophys., 4, 101-124, 1997,

SRef-ID: 1607-7946/npg/1997-4-101.

Nigro, G., Malara, F., Carbone, V., and Veltri, P.: Nanoflares and MHD Turbulence in Coronal Loops: A Hybrid Shell Model, Phys. Rev. Lett., 92, 194501, 1-4, 2004.

Parker, E. N.: Nanoflares and the solar X-ray corona, Astrophys. J, $330,474-479,1988$
Politano, H. and Pouquet, A.: Model of intermittency in magnetohydrodynamic turbulence, Phys. Rev. E, 52, 636-641, 1995.

Priest, E. R.: Solar Magnetohydroynamic, D. Reidel Publishing Company, Dordrecht, 1982.

She, Z.-S., Jackson, E., and Orszag,S. A.: Intermittent vortex structures in homogeneous isotropic turbulence, Nature, 344, 226228, 1990.

She, Z.-S. and Leveque, E.: Universal scaling laws in fully developed turbulence, Phys. Rev. Lett., 72, 336-339, 1994.

Sorriso-Valvo, L., Carbone, V., and Veltri, P.: Intermittency in the Solar Wind Turbulence through probability distribution functions of fluctuations, Geophys. Res. Lett., 26, 1801-1804, 1999.

Strauss, H. R.: Nonlinear, three-dimensional magnetohydrodynamics of noncircular tokamaks, Phys. Fluids, 19, 134-140, 1976.

Tu, C. Y., Marsch, E., and Rosenbauer, H.: An extended structure function model and its aplication to the analysis of solar wind intermittency properties, Ann. Geophys., 14, 270-285, 1996, SRef-ID: 1432-0576/ag/1996-14-270.

Veltri, P, and Mangeney, A.: Scaling Laws and Intermittent Structures in Solar Wind MHD Turbulence in Solar Wind Nine, Edited by Habbal, S. R., Esser, R., Hollweg, J. W., and Isemberg, P. A., AIP Conference Proceedings, AIP Press, New York, USA, 471, 543-546, 1999.

Zank, G. P. and Matthaeus, W. H.: The equations of reduced magnetohydrodynamics, J. Plasma Phys., 48, 1, 85-100, 1992. 